\title{
Discordance in the diagnosis of chronic kidney disease between serum creatinine determination and glomerular filtration rate (calculated by Cockcroft-Gault) in hypertensive and / or diabetic patients in a spray of Araguari-MG
}

\section{Discordância no diagnóstico da doença renal crônica entre dosagem de creatinina sérica e taxa de filtração glomerular (calculada por Cockcroft-Gault) em pacientes hipertensos e/ou diabéticos em uma esf de Araguari-MG}

DOI: $10.46919 / \operatorname{archv} 1 \mathrm{n} 6-004$

Recebimento dos originais: 01/09/2020

Aceitação para publicação: 30/10/2020

\author{
Amanda Vieira Arruda \\ Graduada em Medicina pelo IMEPAC \\ Instituição: Instituto Master de Ensino Presidente Antonio Carlos \\ E-mail: dra.amandava@gmail.com
}

Endereço: Avenida Minas Gerais 1889- Centro Araguari, MG CEP 38440-148

\section{Gabriela Cristina de Alvarenga Araújo}

Graduada em Medicina pelo IMEPAC

Instituição: Instituto Master de Ensino Presidente Antonio Carlos

Endereço: Avenida Minas Gerais 1889- Centro Araguari, MG CEP 38440-148

E-mail: gabiaraujo90@hotmail.com

\section{Gustavo Prado Pouzas Guedes}

Graduado em Medicina pelo IMEPAC

Instituição: Instituto Master de Ensino Presidente Antonio Carlos

Endereço: Avenida Minas Gerais 1889- Centro Araguari, MG CEP 38440-148

E-mail: gpradopouzas@gmail.com

\section{Marilia Borges Carneiro}

Graduada em Medicina pelo IMEPAC

Instituição: Instituto Master de Ensino Presidente Antonio Carlos

Endereço: Avenida Minas Gerais 1889- Centro Araguari, MG CEP 38440-148

E- mail: mariliaborges_ita@hotmail.com

\section{Samuel Ribeiro Dias}

Graduado em Medicina pelo IMEPAC

Instituição: Instituto Master de Ensino Presidente Antonio Carlos

Endereço: Avenida Minas Gerais 1889- Centro Araguari, MG CEP 38440-148

E-mail: sam_rdw@hotmail.com

\section{RESUMO}

A hipertensão arterial (HA) é um problema de saúde pública mundial e um dos principais fatores de risco para o desenvolvimento da doença renal crônica. A HA pode ser tanto a causa como a consequiência de uma doença renal. A DRC pode ocorrer em mais de $75 \%$ dos hipertensos de qualquer idade (BASTOS, 2010) 
O Diabetes mellitus (DM) é uma condição que ocorre quando o corpo não fabrica insulina suficiente ou não consegue utilizar de forma adequada quantidades normais de insulina (hormônio que regula a quantidade de glicose no sangue). Os pacientes diabéticos apresentam risco aumentado para DRC e devem ser monitorizados frequentemente para a ocorrência da lesão renal. O acometimento renal pela DM representa a principal complicação microvascular da doença e a maior causa de insuficiência renal terminal em todo o mundo, cursando com morbidade e mortalidade elevadas. $\mathrm{O}$ risco de desenvolvimento de nefropatia é de cerca de $30 \%$ nos diabéticos tipo 1 e de $20 \%$ nos diabéticos tipo 2. (Junior, 2004) O DM é a principal causa de IRC em pacientes ingressando em hemodiálise (HD) nos países desenvolvidos, representando até quase $50 \%$ dos novos casos. (BURMEISTER, 2012)

O presente trabalho tem por objetivo relatar as vivências no desenvolvimento do tema Metodologia Ativa por meio da utilização do Arco de Charles Maguerez em uma Unidade Básica de Saúde da Família (UBSF) do bairro Miranda em Araguari-MG, durante a disciplina Internato em Sistemas Municipais de Saúde da Unidade de Ensino do Curso de Medicina do Instituto Master de Ensino Presidente Antônio Carlos - IMEPAC.

O Arco de Charles Maguerez é uma das estratégias de ensino-aprendizagem para o desenvolvimento da Problematização e partir da realidade social.

Durante o estudo e a observação da realidade local foram avaliados 487 prontuários, onde nos deparamos com 149 hipertensos, 68 diabéticos, 270 hipertensos e diabéticos. Identifica - se que não ocorreu a avaliação necessária a estes portadores já que de acordo com os protocolos oficiais de HAS e da Sociedade Brasileira de Nefrologia orientam, a investigação clínica e laboratorial anual com a dosagem da creatinina sérica e o cálculo da TFG com o uso da equação de Cockcroft-Gault. Propõe-se a investigação da não aplicabilidade dos protocolos oficiais

Trata-se de um estudo transversal realizado em uma ESF de Araguari-MG. Houveram dois instrumentos de coleta, o primeiro foi a avaliação da Pressão Arterial (PA) realizada um uma reunião de Hipertensos, e o segundo deu-se com a análise do prontuário do grupo de pacientes estudado. Foi considerado como Hipertensão Arterial Sistêmica Controlada níveis menores que 140/90mmhg.

Palavras-Chave: Hipertensão Arterial Sistêmica, Doença Renal Crônica, Diabetes Mellitus, Lesão renal, Filtração glomerular.

\begin{abstract}
Arterial hypertension ( $\mathrm{AH})$ is a worldwide public health problem and one of the main risk factors for the development of chronic kidney disease. AH can be both the cause and the consequence of kidney disease. CKD can occur in more than $75 \%$ of hypertensive patients of any age (BASTOS, 2010)

Diabetes mellitus (DM) is a condition that occurs when the body does not manufacture enough insulin or is unable to properly use normal amounts of insulin (a hormone that regulates the amount of glucose in the blood). Diabetic patients are at increased risk for CKD and should be monitored frequently for kidney damage. Renal involvement by DM represents the main microvascular complication of the disease and the biggest cause of end-stage renal failure worldwide, leading to high morbidity and mortality. The risk of developing nephropathy is about 30\% in type 1 diabetics and 20\% in type 2 diabetics. (Junior, 2004) $\mathrm{DM}$ is the main cause of CRF in patients undergoing hemodialysis (HD) in developed countries, representing up to almost 50\% of new cases. (BURMEISTER, 2012)

The present work aims to report the experiences in the development of the Active Methodology theme through the use of the Charles Maguerez Arch in a Basic Family Health Unit (UBSF) in the Miranda district in Araguari-MG, during the Internship in Municipal Systems discipline Health Unit of the Medical School Teaching Unit of the Instituto Presidente de Ensino Presidente Antônio Carlos IMEPAC.

The Arch of Charles Maguerez is one of the teaching-learning strategies for the development of problematization and take place from the social reality.
\end{abstract}


During the study and observation of the local reality, 487 medical records were evaluated, where we found 149 hypertensive, 68 diabetic, 270 hypertensive and diabetic patients. It is identified that the necessary evaluation for these patients has not occurred since, according to the official protocols of SAH and the Brazilian Society of Nephrology, annual clinical and laboratory investigation with the measurement of serum creatinine and the calculation of GFR with the use of the Cockcroft-Gault equation. The investigation of the non-applicability of official protocols is proposed

This is a cross-sectional study carried out in an ESF in Araguari-MG. There were two collection instruments, the first was the Blood Pressure (BP) assessment carried out at a meeting of Hypertensive Patients, and the second occurred with the analysis of the medical records of the group of patients studied. It was considered as Controlled Systemic Arterial Hypertension levels below 140 / 90mmhg.

KeyWords: Systemic Arterial Hypertension, Chronic Kidney Disease, Diabetes Mellitus, Kidney injury, Glomerular filtration.

\section{INTRODUÇÃO}

A doença renal crônica (DRC) é definida por lesão renal (proteinúria persistente) caracterizada por alterações estruturais ou funcionais dos rins com ou sem redução da taxa de filtração glomerular (TFG).A TFG é a melhor medida do funcionamento renal em indivíduos normais ou pacientes com doença renal. Seu nível varia com a idade, sexo e massa muscular. TFG menor que $60 \mathrm{~mL} / \mathrm{min} / 1,73 \mathrm{~m}^{2}$ representa diminuição de cerca de $50 \%$ da função renal normal e, abaixo deste nível, aumenta a prevalência das complicações da DRC (BASTOS, 2010).

A hipertensão arterial (HA) é um problema de saúde pública mundial e um dos principais fatores de risco para o desenvolvimento da doença renal crônica. A HA pode ser tanto a causa como a conseqüência de uma doença renal. A DRC pode ocorrer em mais de $75 \%$ dos hipertensos de qualquer idade (BASTOS, 2010)

No Brasil, dentre 2.467.812 pacientes com hipertensão e/ou diabetes cadastrados no programa HiperDia do Ministério da Saúde em 29 de março de 2004, a freqüência de doenças renais foi de 6,63\% (175.227 casos). A frequência de DRC na ESF - Miranda II em Araguari-MG foi de 23,1\%, quando considerada a TFG $<60 \mathrm{~mL} / \mathrm{min}(\mathrm{n}=487)$.Portanto, todo paciente hipertenso elou diabético possuem elevado risco para desenvolverem a DRC e devem ser submetidos a exames para averiguar a presença de lesão renal (análise de proteinúria) e para estimar o nível de função renal (TFG) a cada ano. (JUNIOR, 2004)

De acordo com Ministério da Saúde (2013), o número de consultas mínimo para um paciente hipertenso que atingiu a meta pressórica ou diabético controlado é de 1/ano; sendo que a periodicidade das mesmas devem ser estabelecidas de acordo com as necessidades individuais. Enquanto que aquele individuo que apresente HAS e DM, associado ou não a doença renal crônica (DRC) deverá se consultar pelo menos 3x/ano. 
A amostra em estudo se caracterizou pela predominância do sexo feminino $(60,2 \%)$ isso provavelmente se deve ao fato de haver maior número de mulheres inscritas no programa HiperDia e ser uma população que busca mais a assistência à saúde. Quanto a faixa etária tivemos predomínio da população idosa ( $\geq 60$ anos), correspondendo a 55,1\%, justificando a susceptibilidade desta quanto ao risco de lesões renais secundárias a doenças crônicas ser maior em pacientes de idade avançada, pois segundo Bastos et al. há diminuição fisiológica da TFG a partir dos 40 anos. Também reflete o processo de transição demográfica pelo qual o Brasil vem passando nas últimas décadas, dado similar foi encontrado por Santos et.al.

Neste estudo, encontrou-se prevalência de $16,4 \%$ de creatinina sérica $\geq 1,2 \mathrm{mg} / \mathrm{dl}$ e de $23,1 \%$ de TGF $<60 \mathrm{~mL} / \mathrm{min}$ (calculado por Cockcroft-Gault), demonstrando uma discordância de 15,3\% no diagnóstico de DRC. Esses achados corroboram com o estudo realizado por SEGURA et.al., cuja prevalência de DRC pelo Cockcroft-Gault foi de 21,5\% enquanto que pela dosagem de creatinina sérica foi de 7,6\%, apresentando uma discordância de 13,9\%; e SANTOS et.al., onde 24,2\% dos pacientes tinham TFG $<60 \mathrm{~mL} / \mathrm{min}$ (calculado por Cockcroft-Gault) e 7,6\% tinham creatinina sérica > 1,2 $\mathrm{mg} / \mathrm{dl}$, evidenciando assim uma discordância de 16,6\%.

A identificação precoce e o manejo adequado da DRC têm sido reconhecidos como importantes medidas para retardar sua progressão. Na prática clínica, a avaliação inicial da função renal costuma ser realizada por meio da dosagem da creatinina plasmática. Essa conduta se deve à simplicidade do método; entretanto, já foi demonstrado que sua normalidade não é sinônimo da ausência de comprometimento da função renal e se mostra um parâmetro relativamente tardio para detecção da lesão.

Independentemente da doença de base, os principais desfechos em pacientes com DRC são as suas complicações (anemia, acidose metabólica, alteração do metabolismo mineral e desnutrição), decorrentes da perda funcional renal e óbito (principalmente por causas cardiovasculares). Estudos recentes indicam que estes desfechos indesejados podem ser prevenidos ou retardados se a DRC for diagnosticada precocemente e as medidas nefro e cardioprotetoras implementadas precocemente. ${ }^{-}$Infelizmente, a DRC é subdiagnosticada e tratada inadequadamente, resultando na perda de oportunidade para a implementação de prevenção primária, secundária e terciária, em parte devido à falta de conhecimento da definição e classificação dos estágios da doença, bem como a não utilização de testes simples para o diagnóstico e avaliação funcional da doença.

\section{PONTOS-CHAVES}

Segundo SANTOS et.al., a menor prevalência da função renal por meio da creatinina sérica pode ser atribuída ao fato de a mesma não ser um método sensível para avaliação função renal na DRC em 
pacientes assintomáticos, tendo em vista que só haverá alteração após perda superior de $50 \%$ da filtração glomerular. Sendo assim isso reforça a importância e a necessidade do cálculo da TFG, já que a não realização do mesmo pode retardar o diagnóstico da doença.

\section{HIPOTÉSES DE SOLUÇÃO}

- Criação de um questionário para rastreio de DRC visando dinamizar o atendimento de pacientes hipertensos e diabéticos nas ESf's a fim de retardar as consequências de lesões renais e ainda, realizar estratificação de risco para melhor manejo desses pacientes.

- A marginalização do DRC pelos profissionais de saúde e a falta de conhecimento no manejo da doença posterga o diagnóstico e tratamento daqueles acometidos pelas complicações da hipofunção renal.

- Avaliar a função renal por meio da solicitação da dosagem de creatinina sérica e realizar o cálculo da TFG pela equação de Cockcroft-Gault nos pacientes hipertensos e diabéticos.

- Busca ativa dos pacientes supra citados para que os mesmos atinjam o número mínimo de consultas anuais preconizadas pelo Ministério de Saúde e assim possuam um seguimento correto de suas patologias.

- Incentivar os profissionais de saúde a registrarem corretamente os resultados de exames solicitados no prontuário bem como o cálculo da TFG e todos os procedimentos realizados durante a consulta médica.

\section{APLICAÇÃO DA REALIDADE}

- Discussão dos dados no cenário prático e acadêmico;

- Propor o uso do questionário padronizado pela Sociedade Brasileira de Nefrologia;

- Identificação dos pacientes não triados;

- Identificação dos pacientes com DRC.

\section{CONCLUSÃO}

O diagnóstico precoce de uma injuria renal crônica nos permite elaborar uma série de medidas que evitam a progressão do declínio da função renal e do desenvolvimento de complicações, principalmente cardiovasculares. Isso, nos permite evitar, ou, retardar a ocorrência de desfechos indesejáveis como complicações cardiovasculares, terapia de substituição renal, alteração do metabolismo mineral, desnutrição e óbito. 
A DRC é um problema de grande relevância e é reconhecida como uma doença complexa que exige múltiplas abordagens no seu tratamento e, estimar o cálculo da taxa de filtração glomerular nos mostra ser uma importante ferramenta na assistência prestada aos pacientes com fatores de risco para desenvolvê-la.

\section{REFERENCIAS}

BASTOS, Marcus Gomes; BREGMAN, Rachel; KIRSZTAJN, Gianna Mastroianni. Doença renal crônica: frequente e grave, mas também prevenível e tratável. Rev. Assoc. Med. Bras., São Paulo , v. 56, n. 2, p. 248-253, $2010 \quad$ Available from $<$ http://www.scielo.br/scielo.php?script=sci_arttext\&pid=S0104-

42302010000200028\&lng=en\&nrm=iso $>$.access on 02 Sept. 2015. http://dx.doi.org/10.1590/S010442302010000200028 .

BURMEISTER, Jayme Eduardo et al .Prevalência de diabetes mellitus em pacientes renais crônicos sob hemodiálise em Porto Alegre, Brasil. J. Bras.Nefrol., São Paulo , v. 34, n. 2, p. 117-121, June 2012. Available from <http://www.scielo.br/scielo.php?script=sci_arttext\&pid=S010128002012000200003\&lng=en\&nrm=iso $>$.access on 10 Sept. 2015. http://dx.doi.org/10.1590/S010128002012000200003.

SANTOS, Elisângela Milhomem dos et al . Valor da equação Cockcroft-Gault na triagem de função renal reduzida em pacientes com hipertensão arterial sistêmica. J. Bras.Nefrol., São Paulo, v. 33, n. 3, p. 313 321, Sept. 2011 . Available from <http://www.scielo.br/scielo.php?script=sci_arttext\&pid=S010128002011000300007\&lng=en\&nrm=iso $>$.access on 02 Sept. 2015. http://dx.doi.org/10.1590/S010128002011000300007

BRASIL. Ministério da Saúde (BR). Secretária de Atenção à Saúde, Departamento de Atenção Básica. Hipertensão arterial sistêmica para o Sistema Único de Saúde. Brasília: Ministério da Saúde; 2006. (BRASIL,2006)

SBC.Sociedade Brasileira de Cardiologia; Sociedade Brasileira de Hipertensão; Sociedade Brasileira de Nefrologia. VI Diretrizes Brasileiras de Hipertensão. Arq Bras Cardiol.2010;95(1 Suppl1):1-51

Junior JER. Doença Renal Crônica: Definição, Epidemiologia e Classificação. J. Bras. Nefrol. 2004;26(3):1-3 